\title{
Nondestructive Evaluation of Changes in Mechanical Properties in Carbon-Carbon Composites during Processing
}

\author{
LEE MoORE AND Ronald Kline \\ School of Aerospace and Mechanical Engineering \\ The University of Oklahoma \\ 865 Asp Ave., Room 212 \\ Norman, OK 73019-0601 \\ ERIC MADARAS AND PHILIP RANSONE \\ NASA/Langley Research Center \\ Hampton, VA 23665 \\ (Received April 13, 1993) \\ (Revised September 7. 1993)
}

\begin{abstract}
In this work, changes in the mechanical properties of carbon-carbon composites during processing are studied using nondestructive characterization techniques. Ultrasonics and radiography are used to measure the local stiffnesses in carbon-carbon panels after each stage in the processing cycle. The degree of material inhomogeneity (as measured by the standard deviation in the local moduli) was monitored throughout the fabrication process. The nondestructively measured stiffnesses were compared with direct mechanical measurements to assess the viability of the technique.
\end{abstract}

\section{INTRODUCTION}

$\mathbf{I}^{\mathbf{2}}$ $\mathrm{N}$ THE NEXT decade, there will be a concerted effort to develop hypersonic aircraft for commercial applications. It is anticipated that the design of these vehicles will place new demands on material performance at elevated temperatures. Temperatures in excess of $3200^{\circ} \mathrm{F}$ will be experienced at hypersonic speeds in certain critical areas of the structure, such as the tip of the nose and the wing leading edges. Carbon-carbon composites are one of the few structural materials available which retain a significant fraction of their specific stiffness and strength at elevated temperatures. Consequently, there is a great deal of interest in carbon-carbon composites as structural materials for hypersonic applications. While this material offers much promise for the future, many problems remain to be solved before it can be effectively utilized.

One of the most important considerations in carbon-carbon development is the effect of processing on the microstructure of the final product. Carbon-carbon 
composites are fabricated in a complicated, multistep process. Initially, a composite panel (called the mold panel in this study) is fabricated using conventional processing techniques. The matrix of this precursor composite is a phenolic resin and graphite fibers are used as reinforcement. The precursor composite is then exposed to elevated temperature $\left(1500^{\circ} \mathrm{F}\right.$ or higher) to convert the resin to pure carbon. After this carbonization step, the material has a very high porosity and poor mechanical properties. To reduce the porosity and improve material properties, the panel is reinfiltrated with resin and returned to elevated temperature for conversion of the new resin into carbon. As this process is somewhat inefficient in filling the sample pores, it must be repeated several times, usually up to four, until a part with reasonable microstructural uniformity is obtained. An alternative to liquid resin infiltration and carbonization is the direct deposition of carbon by means of chemical vapor infiltration; however, this process also usually requires two or more repetitions and may involve interim machining and heat treatments for graphictization. Clearly, the properties of the final composite are heavily dependent on the efficiency of the pore filling process.

In this research effort, we examine the use of quantitative, nondestructive testing techniques to track changes in the properties of carbon-carbon composites during processing. Here, we use a combined radiography/ultrasonic velocity approach to characterize the behavior of the composite on a local basis. As wave speed is a function of both moduli and density, both nondestructive evaluation techniques are needed for accurate moduli reconstruction in a porous media such as carbon-carbon. In order to demonstrate the validity of the approach, we compare our results with direct mechanical property measurements as well as photomicrographs of the specimen microstructure taken at various stages of processing.

\section{BACKGROUND}

A variety of ultrasonic test techniques have been developed for quantitative characterization of the mechanical properties of composite media. Much of the early work in this area involved sectioning of the composite to generate the required waves for elastic moduli reconstruction [1]. However, the value of using nondestructive, oblique incidence ultrasonic techniques for this determination was also recognized [2]. More recently, a plethora of approaches have emerged based on bulk wave slowness surface reconstruction [3], point source-point sensor techniques [4], surface wave analysis [5], critical angle measurements [6], and plate wave propagation - the so-called leaky-Lamb wave method [7]. The key requirement for any viable NDE application of any of these approaches is the capability for rapid, local property determination so large scale parts can be scanned in a timely fashion.

While many of these approaches have been developed for conventional laminated composites, relatively little work in this area has been done on carboncarbon materials. The one major exception to this observation is the work of Hosten and Tittman [8], who examined the effect of processing on the elastic moduli of woven carbon-carbon composite samples. Even though these researchers examined only a limited number of moduli in the orthotropic composite, the 
authors clearly demonstrate the viability of modulus reconstruction technique to study processing induced changes in the composite.

\section{THEORY}

The governing equations for wave propagation in anisotropic media are relatively straightforward. Assuming plane wave propagation of the form

$$
u_{j}=A_{o} \alpha_{j}^{e i\left(k l_{i} x_{i}-w t\right)}
$$

the equations of motion reduce to the following eigenvalue problem

$$
(\underset{\tilde{\alpha}}{\lambda}-\underset{\tilde{z}}{I}) \underset{\alpha}{\alpha}=0
$$

where

$$
\begin{aligned}
\lambda_{i k} & \equiv C_{i j k l} m_{j} m_{l} / \varrho \\
I & =\text { identity matrix } \\
\varrho & =\text { density } \\
\underline{m} & =\underline{l} \mathbf{v}=\text { slowness vector } \\
l & =\text { wave normal direction cosines } \\
\hat{k} & =\text { wave number } \\
\mathbf{V} & =\text { phase velocity } \\
\alpha & =\text { direction cosines of particle displacements } \\
C_{i j k l} & =\text { elastic-stiffness tensor }
\end{aligned}
$$

For material property characterization, we are particularly interested in velocity. In any given direction $(l)$, velocity will be a function of the moduli and density. If a sufficient number of independent velocities can be measured, there will in principle be sufficient equations to determine each of the elastic moduli. For an orthotropic media, nine moduli are sought.

A variety of approaches have been utilized to obtain the measurements needed for modulus reconstruction. Mignogna et al. [9] use an oversampling approach with 27 measurements to reconstruct the nine desired moduli. Hosten et al. [10] and Kline et al. [11] use a direct approach (with no redundancy) with the minimum nine measurements. This approach takes optimal advantage of the material symmetry to reduce the complexity of the problem. First, the through thickness transit time is determined to yield $C_{33}$ directly. Next, with the transducer axis oriented in the zero degree direction, the Christoffel equation assumes a relatively simple form with contributions only from the known $C_{33}$ term and three unknown $C_{13}, C_{11}$ and $C_{55}$. These three terms are obtained from measurements of the transit time for one quasilongitudinal wave ( $5^{\circ}$ angle of incidence) and two quasitransverse waves $\left(15^{\circ}\right.$ and $20^{\circ}$ angles of incidence). Rotating the transducer $90^{\circ}$, and repeating exactly the same procedure isolates three additional moduli $C_{23}, C_{22}$ and $C_{44}$. The remaining two moduli are obtained with the transducers oriented at $45^{\circ}$ with respect to the symmetry axes. The resulting Christoffel equa- 
tion includes all nine constants (the seven previously determined and the remaining unknowns $C_{12}$ and $C_{66}$ ); hence any errors in the first measurements will be reflected in the determination of $C_{12}$ and $C_{66}$.

Also it should be pointed out that, in anisotropic media, the phase and group velocities do not coincide. Since group (not phase) velocity is the quantity being measured, we modify the problem so that group velocity is properly treated. Group and phase velocities are related via the equation:

$$
S_{q}=\frac{C_{i j k q} \mathbf{1}_{j} \alpha_{k} \alpha_{i}}{\varrho \mathbf{v}}
$$

where $|S|=\nu_{\text {group }}$. This relationship is a little more complicated algebraically than simply solving the Christoffel equation directly, but otherwise readily tractable mathematically using standard, nonlinear equation solving algorithms. A more detailed discussion of alternative strategies to modulus reconstruction in orthotropic media may be found in Reference [12].

\section{EXPERIMENTAL PROCEDURE}

\section{Test Sample Fabrication}

To illustrate the capability of our quantitative nondestructive evaluation techniques, we recently studied a series of C-C test panels to investigate the effects of processing on material properties. The processing of these samples was stopped at different stages in the manufacture in order to examine the effectiveness of the reinfiltration process. The carbon fiber prepreg (phenolic resin and carbon fibers) was cut into square sheets for assembly into an eight-layer, cross-ply composite plate. The prepreg was a woven 8-harness satin fabric of 3K T-300 fiber tows with a warp of 24 tows per inch and a fill of 23 tows per inch. To ensure a tight fit between each of the laminae, the prepreg sheets were positioned so that the warp side of each of the laminae was facing up and the fill side down; thus, the laminae fit more closely together producing a higher fiber volume.

The laminae was vacuum bagged and molded in a press using a stepped incremental heating ramp to $325^{\circ} \mathrm{F}$ over a four and one-half hour time period. The total pressure of consolidation during mold/cure was $45 \mathrm{psi}(15 \mathrm{psi}$ bag $+30 \mathrm{psi}$ press).

Once the mold/cure process was accomplished, the two plates were cut into smaller panels. With the first step completed in the manufacturing process one panel was marked as mold/cure and kept as the first in the series of seven panels representing the processing steps under investigation. The remaining seven panels went into the first pyrolyzation which heated the $\mathrm{C}-\mathrm{C}$ panels to $1600^{\circ} \mathrm{F}$ to complete the carbonization of the phenolic resin. At the end of the first pyrolyzation a second panel was retained and designated $\left(\mathrm{P}_{0}\right)$ for zero densifications. The remaining six panels went onto the first densification; this involved immersing the panels in liquid resin, heating to $176^{\circ} \mathrm{F}$ under a vacuum until the air was boiled out, and then pressurizing the liquid and panels to $180 \mathrm{psi}$ for one hour. 
Once the panels were cooled down they were wiped down to remove excess resin and cured to $325^{\circ} \mathrm{F}$ before being pyrolyzed again.

It was after the second pyrolyzation that the third panel was retained and designated $\left(\mathrm{P}_{1}\right)$ for one densification. The remaining panels continued through the densification/pyrolyzation process yielding the $\left(\mathrm{P}_{2}, \mathrm{P}_{3}, \mathrm{P}_{4}\right.$, and $\left.\mathrm{P}_{5}\right)$ panels for the remaining tests. The sample panels were then coated with an encapsulator to keep water from penetrating the pores of the material during the immersion testing.

\section{Ultrasonic Testing}

The experimental setup for ultrasonic property determination is illustrated in Figure 1. Here, a through transmission arrangement was used to minimize signal loss in the porous carbon-carbon samples. Also, as stated previously, samples were treated with a very thin polymer coating to prevent moisture uptake by the sample. A matched pair of $1 \mathrm{MHz}$ center frequency, resonant transducers were used to perform the ultrasonic inspection. In order to enhance the accuracy of the time delay measurements, a pulse phase locked loop system was used. This allowed us to make extremely accurate measurements of the changes in the arrival times of the appropriate acoustic signals. Since this procedure yields relative, not absolute, it was necessary to establish an accurate set of reference measurements at the initial starting point. This was done using a crosscorrelation technique to compare the transit time between sensors both with and without the sample inserted between them. In this way, we were able to scan the parts rapidly for each inspection angle without sacrificing accuracy. As described in the previous section, nine separate scans were required for the reconstruction of the nine orthotropic moduli.

\section{Density Measurements}

Radiographic test methods were used for local density determination in the composite samples. For this measurement, radiographs of the complete parts were made along with an X-ray image of an aluminum calibration bar (stepped in increments of 0.05 inch). Then, we converted the photographic x-ray images to digital form. This was done using a digitizing camera. By comparing the $\mathrm{C}-\mathrm{C}$ calibration block to the Al sample, the absorption coefficient for $\mathrm{C}-\mathrm{C}$ was then directly established. This permitted the local density measurements needed for modulus reconstruction.

\section{Mechanical Property Measurements}

In order to verify the accuracy of the ultrasonic test procedure, mechanical property measurements were conducted on samples cut from the test panels. Test coupons were cut at $0^{\circ}, 45^{\circ}$ and $90^{\circ}$ with respect to the warp/fill directions of the fiber reinforcement. Samples were instrumental with strain gages prior to testing. The ends of the samples were then potted in resin. When sample testing was complete, the test coupons were subjected to compressive loading. In this 


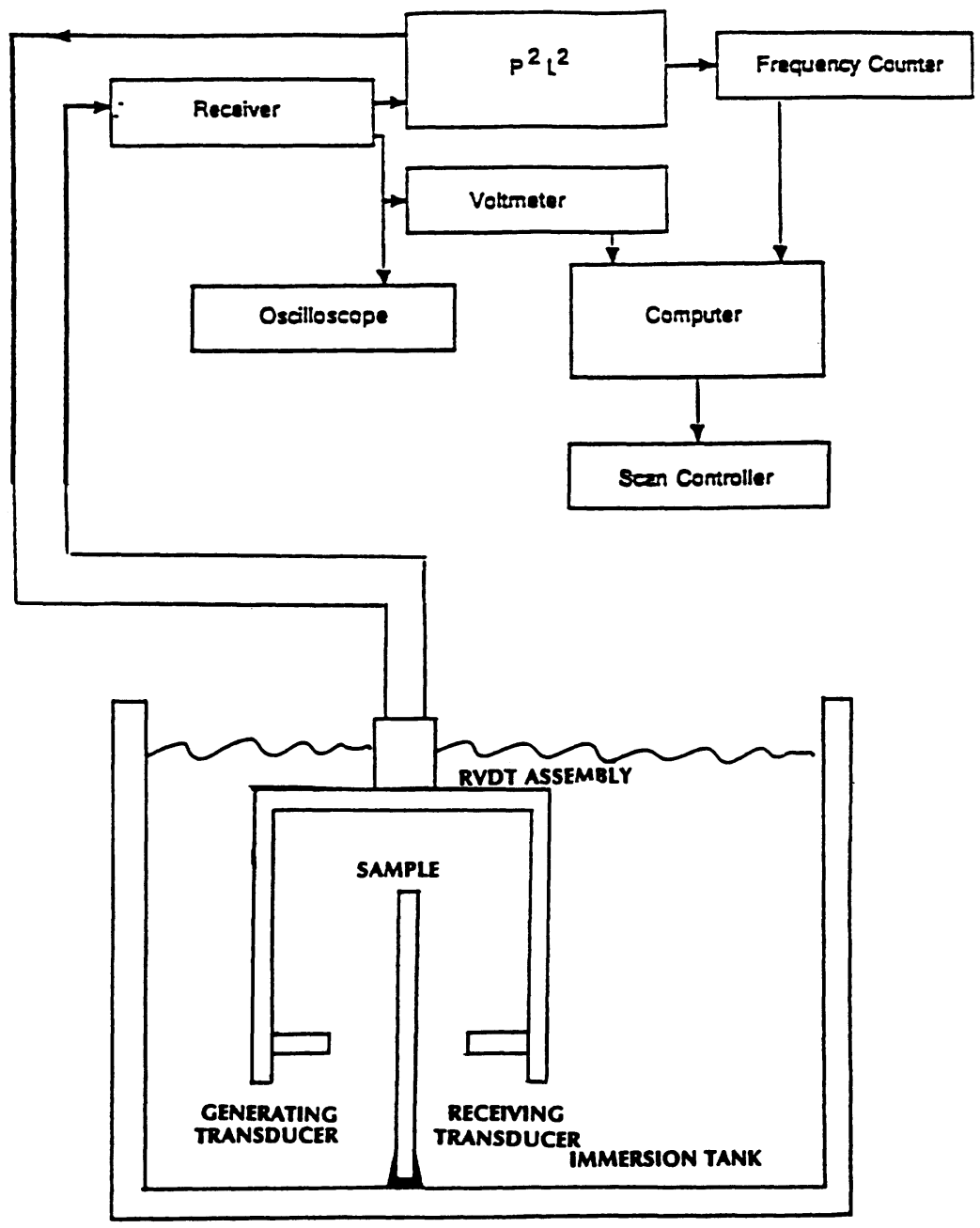

Figure 1. Experimental geometry.

way it was possible to experimentally determine 7 of the 9 orthotropic moduli. The limited sample thickness prevented direct determination of the remaining two moduli.

\section{RESULTS AND DISCUSSION}

Ultrasonic test techniques were used to determine the nine pertinent moduli for the orthotropic samples. Results were then compared with mechanical test results for coupon cut from the plates. Average porosity was also determined for each 
sample plate. Results are presented in Figure 2 . As expected, the average porosity was found to increase dramatically with the first pyrolization and then slowly decrease with successive densifications. Changes in pore size with processing were studied previously at NASA/Langley Research Center [13]. Figure 3 shows that the successive densifications of the manufacturing process preferentially fill the 0.3 - to 10 -micrometer-sized pores and that large pores do not fill well.

To examine this further, representative photo micrographs were obtained for each of the sample densifications. Representative micrographs are presented in Figure 4. Several major microstructural defects are found in the micrographs: matrix porosity (principally seen at fiber crossing points), fiber-matrix debonding and matrix microcracking which both occur as a result of matrix shrinkage during pyrolysis. As seen in the photo micrographs, large scale porosity is only reduced slightly by successive reinfiltrations. By the final step, the small scale porosity and matrix microcracking are reduced substantially from that of the $\mathrm{P}_{0}$ state, but not eliminated. Furthermore, fiber matrix debonding which was virtually complete throughout the specimen in the $\mathrm{P}_{0}$ state, is also improved by the $\mathrm{P}_{5}$ densification, but also not completely eliminated.

For the ultrasonic method to be meaningful, the mechanical test values, although obtained from multiple test coupons spread out over the surface of the six sample panels, should be representative of the values obtained by the ultrasonic wave velocity analysis. The data shown in Figure 5 exhibit the relationship between the compressive test and ultrasonic stiffness values for selected moduli. The upper and lower ultrasonic stiffness values are shown on the graphs so that the entire range of stiffness values obtained over the sample panel's surface may

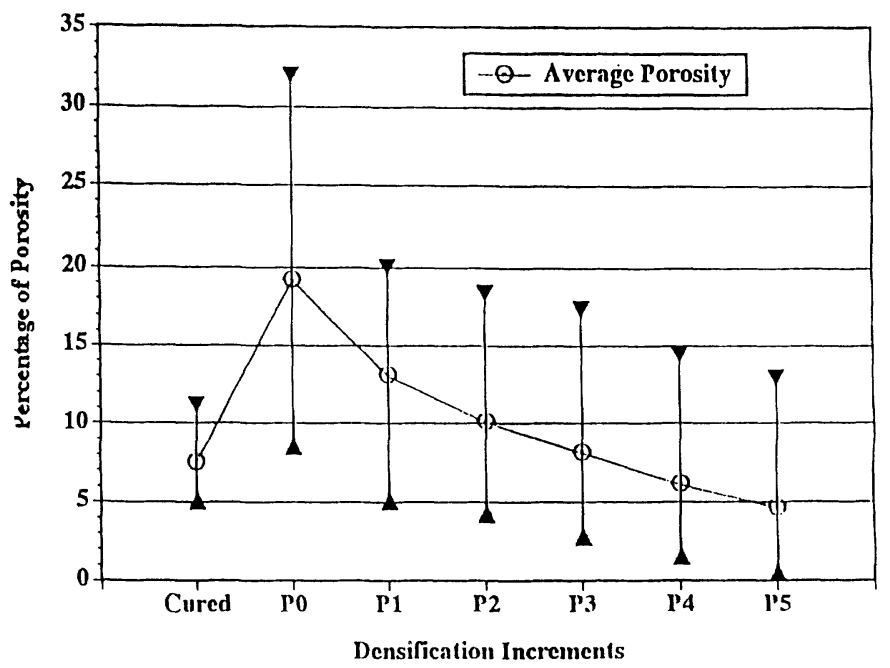

Figure 2. Effect of processing history on C-C composite panel porosity. 


\section{TYPICAL EVOLUTION OF ACC PORE DISTRIBUTION WITH SUCCESSIVE DENSIFICATION}

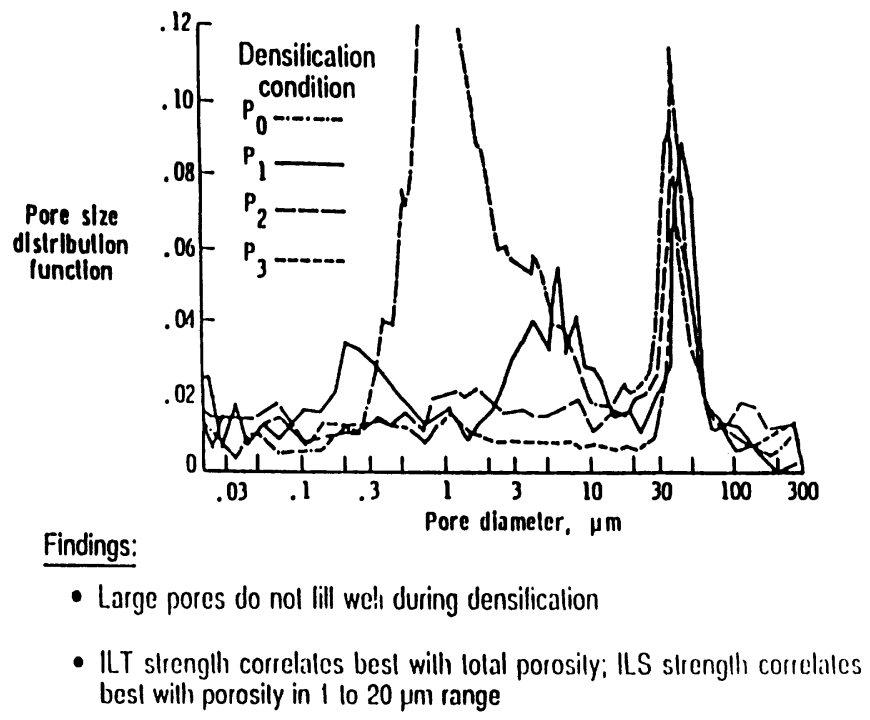

Figure 3. Effect of processing history on coated C-C composite pore site distribution (after Maahs [9]).

be represented in the comparison.* It should be noted that the ultrasonic values for each panel represent 121 individual measurements $[11 \times 11$ grid size, determined by panel size and sensitive area of transducer $\left.\left(.375^{\prime \prime}\right)\right]$.

Three important observations may be made from these data. The first is that for each of the seven stiffness coefficients investigated, the mechanical test values were within the high-low spread of ultrasonic test values. This clearly implies that the ultrasonic stiffness values were reliable predictions of the material stiffness. The one exception was the $\mathrm{P}_{\mathbf{o}}$ panel in which the mechanical test values were either at the low end or out of range of the ultrasonic stiffness values. This anomaly in the accuracy of the ultrasonic method may best be explained by the high void-porosity value found in the $\mathrm{P}_{\mathrm{o}}$ panel.

Secondly, there is a very large degree of data scatter within a sample. This is best illustrated in Figure 6 where the standard deviation for several modulus measurements is presented as a function of processing history. The degree of material inhomogeneity is particularly pronounced in the $P_{0}$ sample, but observed in all samples. This result is expected due to the large amount of poros-

*Typically, the ultrasonic values were more or less uniformly distributed about the midpoint between the high and low values. It should be noted that differences between the average ultrasonic values and the mechanical measurements are to be expected since the mechanical test samples were cut from a small section at the entire panel. 

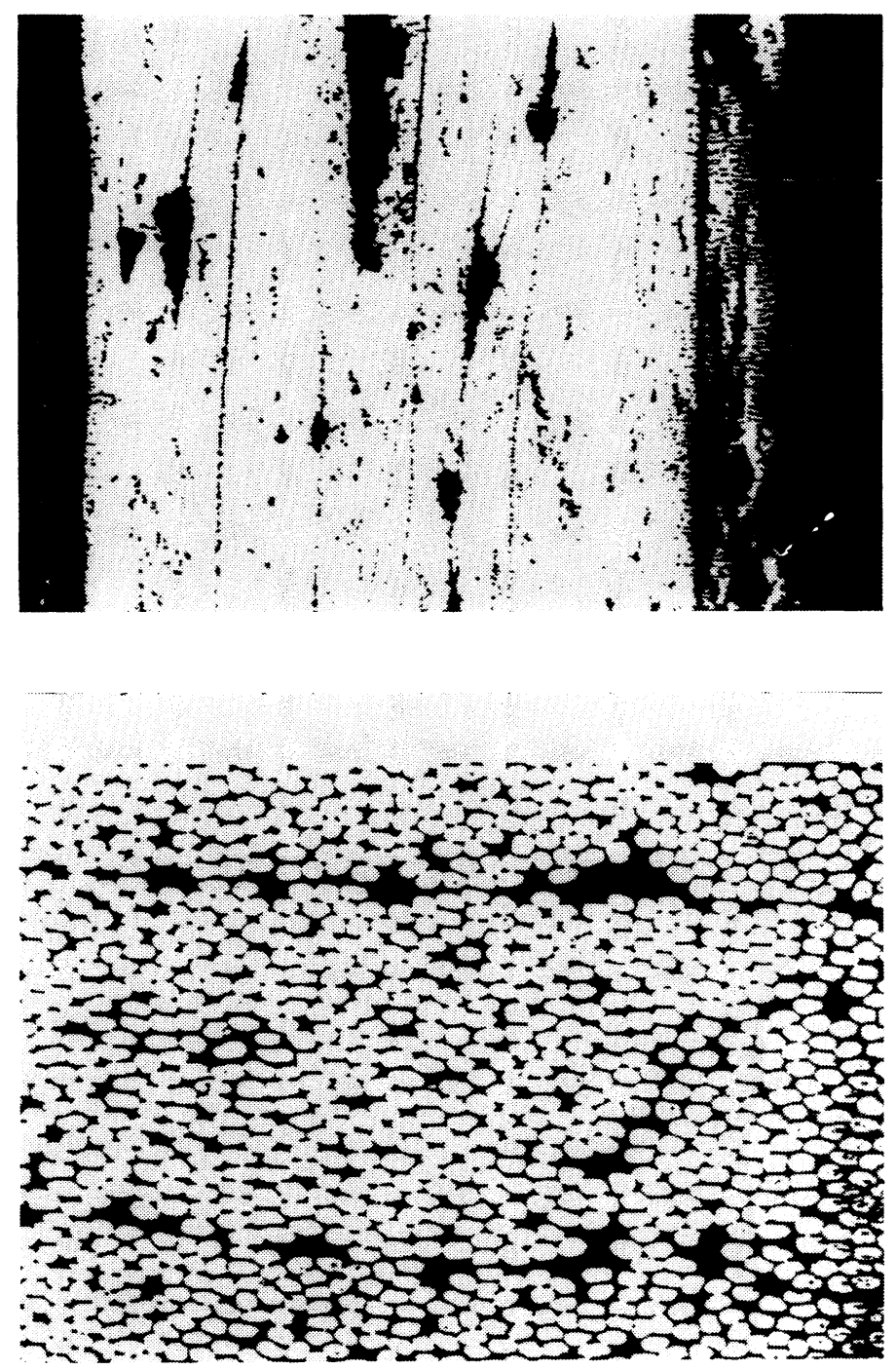

Figure 4a. Void-porosity (top) and microcracking (bottom) found in the mold/cure sample panel. Note, no microcracking at this processing step. Average porosity $+7.64 \%$. 

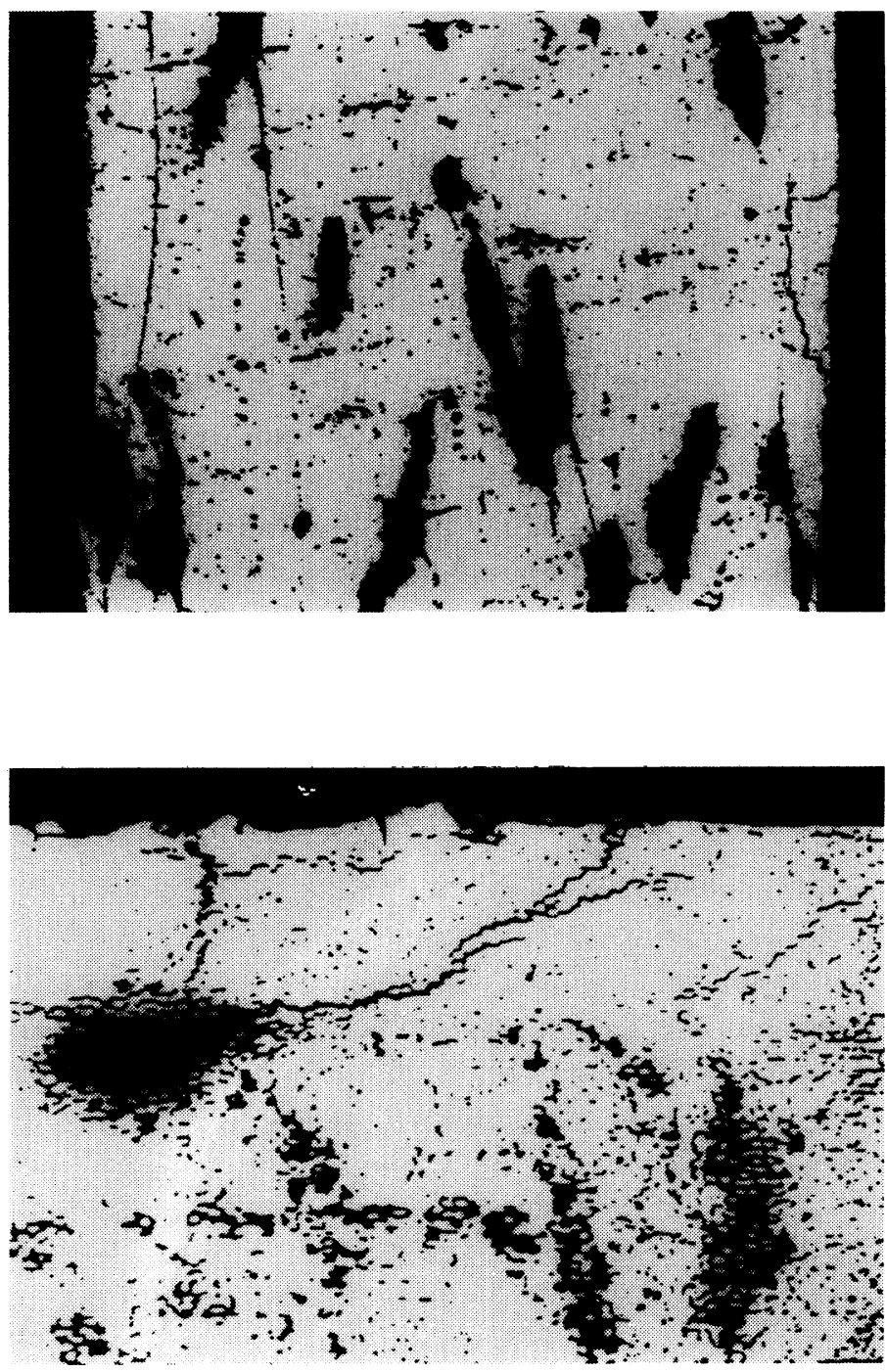

Figure 4b. Void-porosity (top) and microcracking (bottom) found in the $P_{1}$ panel. Average porosity $+15.43 \%$. 

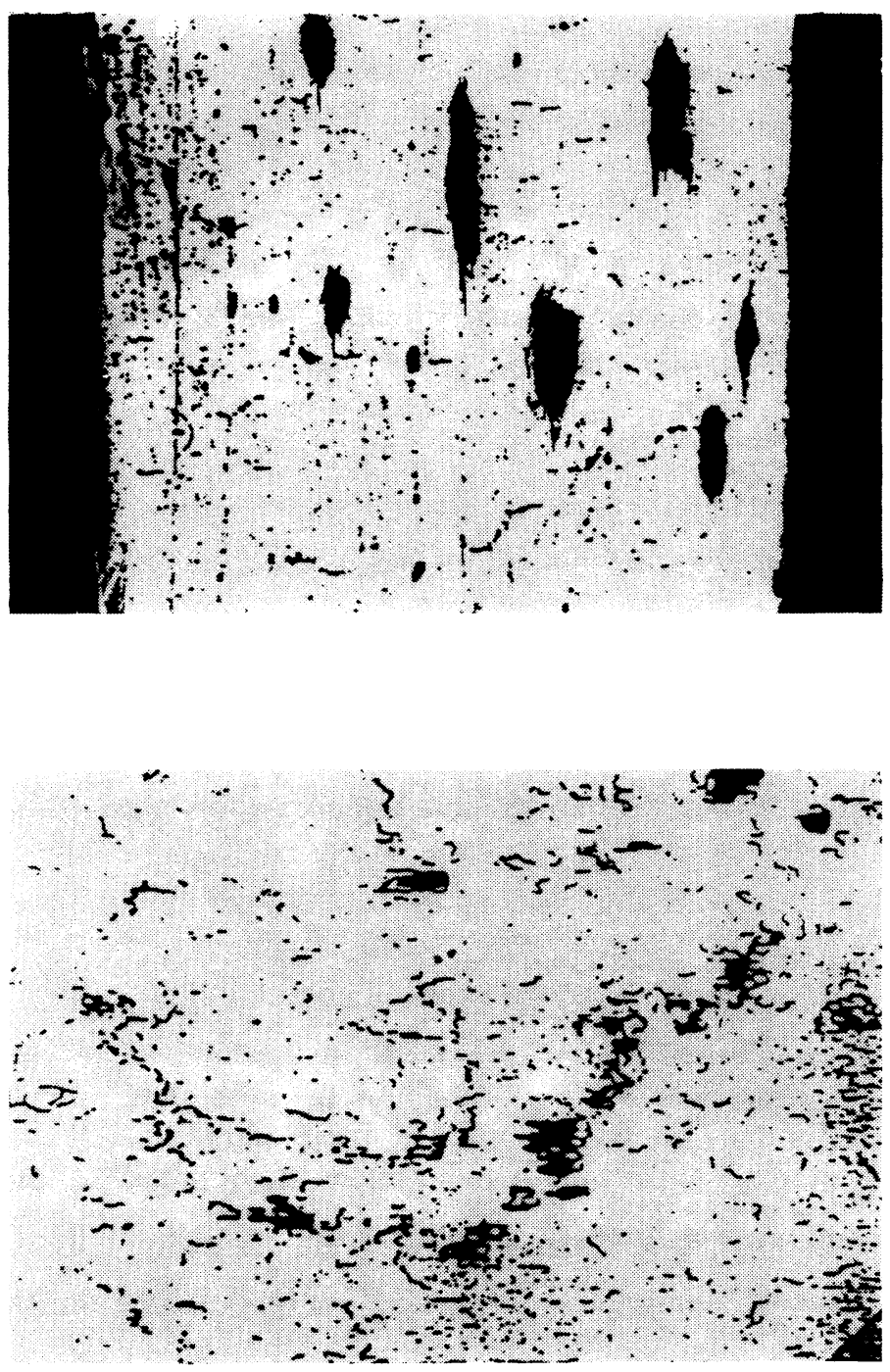

Figure 4c. Void-porosity (top) and microcracking (bottom) found in the $P_{4}$ panel. Average porosity $=7.53 \%$. 


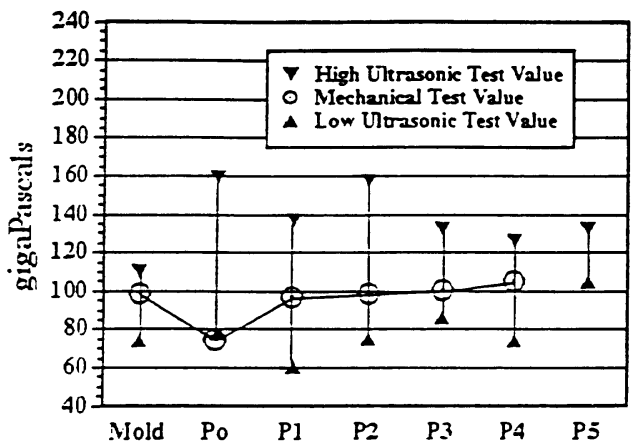

Figure 5a. Ultrasonic and compressive test values of the $C_{11}$ stiffness coefficient.

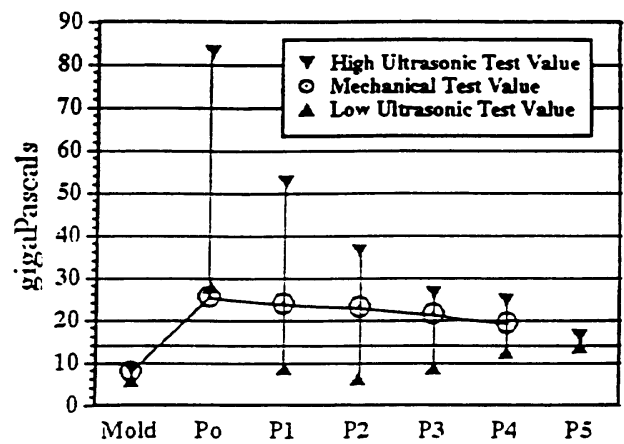

Figure 5b. Ultrasonic and compressive test values of the $C_{12}$ stiffness coefficient.

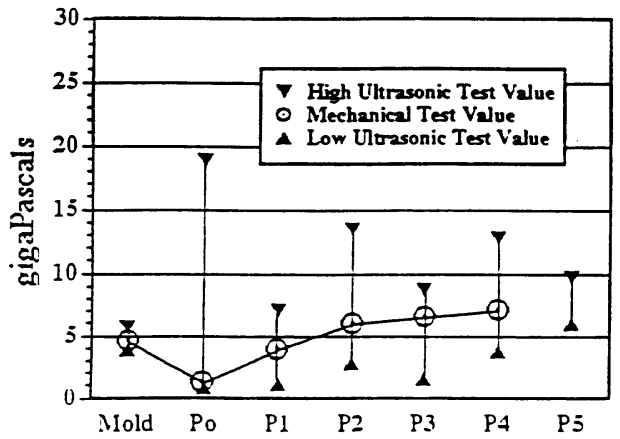

Figure 5c. Ultrasonic and compressive test values of the $C_{66}$ stiffness coefficient. 


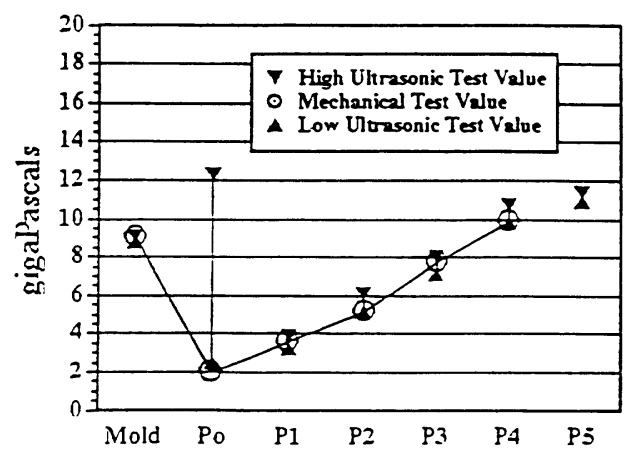

Figure 5d. Ultrasonic and compressive test values of the $C_{33}$ stiffness coefficient.

ity present in the samples. However, it should be noted that the degree of inhomogeneity present in the sample (as measured by the standard deviation in the local moduli) decreases with increased processing beyond the $\mathrm{P}_{0}$ state. In fact, the degree of inhomogeneity, observed after five processing cycles, is comparable to that of the initial composite.

The third observation is the effect of the manufacturing processing (densification) on the individual stiffness components. As observed in the mechanical test

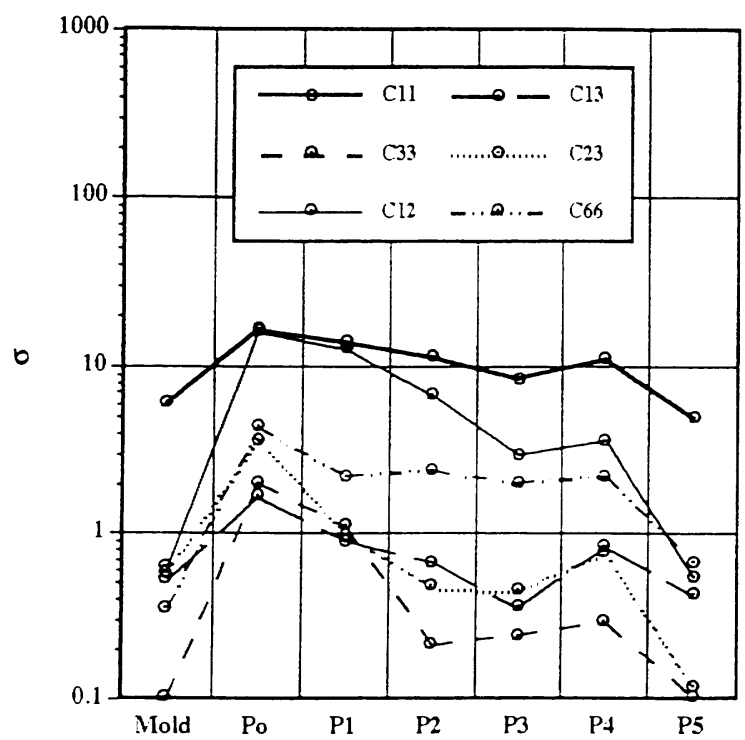

Figure 6. Standard deviation of ultrasonic stiffness values through the seven manufacturing steps. 
results, the ultrasonic stiffness values generally decreased with the first pyrolyzation and then increased with each successive densification. This is again a result of the manufacturing process.

Clearly, considerable damage, hence material property degradation, is introduced into the sample driving the initial processing stage $\left(\mathrm{P}_{0}\right)$, of particular interest is the observation that this effect is most prominent for the matrixdominated properties. For example, the normal stiffness in two fiber directions $C_{11}$ and $C_{22}$ are only reduced to about $20 \%$ from their initial (phenolic composite) value, while the through-thickness normal stiffness is reduced to $25 \%$ of its initial value. Thus, even though the fibers are almost completely debonded from the matrix, there is only a marginal reduction in the normal stiffness (in-plane) as the matrix carries very little of the load. For moduli which depend heavily on the transfer of load between fiber and matrix, the reduction is significantly more pronounced. One modulus $C_{12}$ was actually found to be stiffer after the initial processing stage. This is a Poisson-type term in the plane of material reinforcement. This modulus was found to decrease with increasing densification. We also find that the normal and shear stiffness were all higher than the values for the original composite. This effect is probably due to the increased stiffness of the matrix. The two remaining moduli $C_{13}$ and $C_{23}$ never returned to their original values. Also, the behavior of these moduli seemed to be asymptotic with processing. Hence, we doubt that further processing will markedly influence the overall composite stiffness. Finally it should be noted that these results apply to stiffnesses, not strength. The large scale defect structure introduced by processing means that extensive strength reduction is to be expected of the final structure.

\section{CONCLUSIONS}

Quantitative nondestructive test techniques have been used to study the effects of processing on the mechanical behavior of carbon-carbon composites.

Most of the elastic moduli (stiffnesses) were found to decrease after the first pyrolization and increase thereafter. In many cases the stiffness values in the final state were higher than they were in the precursor composite. Poisson type terms $\left(C_{12}, C_{13}, C_{23}\right)$ behaved in an opposite fashion. The degree of material inhomogeneity was found to decrease with each processing stage.

Results were compared with direct mechanical property measurements, good agreement was observed.

A photomicrographic study of changes in material microstructure was also completed. While overall sample porosity decreased with processing, small scale pores were found to fill preferentially. Also, matrix microcracks were not found to fill efficiently in the impregnation/pyrolization process.

From these observations, we conclude that the increase in stiffness with processing is attributable to the increased stiffness of the carbon matrix over its polymer precursor. We also believe that part quality would be considerably increased if a more uniform pore size distribution could be achieved in the initial pyrolization. 


\section{REFERENCES}

1. Kriz, R. and W. Stinchcomb. 1979. "Elastic Moduli of Transversely Isotropic Graphite Fibers and Their Components," Experimental Mechanics, 19:41-49.

2. Markham, M. F. 1970. "Measurement of the Elastic Contents of Fiber Composite by Ultrasonics," Ultrasonics, pp. 145-149.

3. Hosten. B., M. Deschamps and B. Tittmann. 1988. "Inhomogeneous Wave Generation and Propagation in Lossy Anisotropic Solids: Application to the Characterization of Viscoelastic Composite Material," J. Acoust. Soc. AM, 82:986-992.

4. Castagnede, B. and W. Sachse. 1988. "Optimized Determination of Elastic Constants from Wavespeed Measurements," Review of Progress in QNDE, 8:1855-1862.

5. Pilaski, A. and J. Rose. 1989. "Use of Subsurface Longitudinal Waves in Composite Material Characterization," Ultrasonics, 27:226-233.

6. Rokhlin, S. and L. Wang. 1989. "Critical Angle Measurement of Elastic Constants in Composite Materials," J. Acoust. Soc. AM, 86:1876-1881.

7. Rokhlin, S. and D. Chimenti. 1990. "Reconstruction of Elastic Constants from Ultrasonic Reflectivity Data in a Fluid-Coupled Composite Plate," Review of Progress in QNDE, 9:1411-1419.

8. Hosten, B. and B. Tittman. 1986. "Elastic Anisotropy of Carbon-Carbon Composites during the Fabrication Process," Proceedings, 1986 Symposium on Sonics and Ultrasonics, IEEE UFFC-34. pp. 1061- 1063 .

9. Mignogna, R. 1991. "Ultrasonic Determination of Elastic Constants from Oblique Angles of Incidence in Non-Symmetry Planes," Review of Process in QNDE, 10B:1669-1675.

10. Houten, B., M. Deschumps and B. T. Tittmann. 1987. "Inhomogeneous Wave Generation and Propagation in Lossy Anisotropic Solids," J. Acoust. Soc. Am., 82:1763-1770.

11. Kline. R.. G. Civse, A. Strit and E. Madures. 1993. "Integrating NDE-Derived Engineering Properties with Finite Element Analysis for Structural Composite Material," 31:53-60.

12. Kline, R. 1992. Nondestructive Characterization of Composite Materials. Lancaster, PA: Technomic Publishing Company, Inc.

13. Maahs, H., P. Ransone and R. Yamaki. 1988. "Evolution of Microstructure, Pore Size and Interlaminar Strength of ACC Type Carbon-Carbon with Successive Densification," NASA TP 2754. 